\title{
Saving Petroleum using Smart Strategies
}

Abha Shukla and Swaranjit Singh Cameotra*

Institute of Microbial Technology, Sector 39 A, Chandigarh 160036, India

"Corresponding author: Swaranjit Singh Cameotra, Senior Principal Scientist, Institute of Microbial Technology, Sector 39 A, Chandigarh 160036, India, Tel: 91 9041036750; E-mail: ssc@imtech.res.in

Received date: April 13, 2015; Accepted date: April 14, 2015; Published date: April 21, 2015

Copyright: (C) 2015 Shukla A, et al. This is an open-access article distributed under the terms of the Creative Commons Attribution License, which permits unrestricted use, distribution, and reproduction in any medium, provided the original author and source are credited.

\section{Introduction}

A large fraction of energy demand is fulfilled by petroleum oil fractions worldwide followed by coal, natural gas, renewable combustible waste, nuclear and hydro energies. Petroleum oil is the backbone of a country to meet its energy demand. However, the imbalance of energy consumption is prevalent around the world due to rapidly depleting energy reserves and escalating oil demand. As petroleum is the most abundant and most highly consumed energy source currently, it is expected that these energy tanks will be empty very soon in near the future. To refill these tanks, there is a need to explore some other energy substitutes or their sources which can meet and compensate energy requirement and energy depletion respectively, in the coming years. Energy demand can be met by exploring new energy sources along with the smart management for oil utilization strategies. Here our concept presented is "The utilization of petroleum oil to a maximum extent as an energy source."

We have limited oil reserves worldwide which are drilled continuously for oil production to the peak. Interestingly a large portion of the petroleum oil remains unused and is wasted due to unavailability of processing technology that is economical. Scientists worldwide are working on the heavy end processing. Although various chemical products are obtained from the crude oil residue but a large amount of oil remains trapped in the oil sludge. We need to develop methods or technologies to convert a large fraction of this end to useful energy source. A large amount of oil loss occurs during processing, for example, oil trapped in oil tank bottom sludge which is not retrieved back, as many physical or chemical methods for the oil recovery are economically or practically unfeasible at large scales. Here smart microbes can be very useful that can help in leaching out the trapped oil in the sludge. A number of microorganisms are known to produce surfactants. It is well established that these microbial surfactants, popularly known as biosurfactants have great affinity towards oil and can be very helpful in oil recovery. Biosurfactants decreases the viscosity of oil during the transportation through pipes which is also a way to decrease the loss of oil during the process. These microbial surfactants are better from economic point of view as the microbes can produces these biosurfactants utilizing cheap substrates like molasses, whey, fruit and vegetable waste or other cheaper carbon sources. Moreover, as biosurfactants have low toxicity they do not raise environmental concerns and have relatively high degradation rates comparative to synthetic surfactants. Biosurfactants are needed in tiny amounts for recovery of oil from sludge. The oil thus recovered can be blended to original crude oil and this can provide high economic returns to the oil industry.

Oil companies can drill out $50-60 \%$ oil and after this no more oil can be pumped out. Oil sticks to the clay, rocks and soil and is very viscous. When a water flood containing biosurfactants is pumped in the abandoned wells, the oil bound is mobilized and can be recovered along with the water as oil-in-water oil emulsions.

Although petroleum is a nonrenewable source of energy and its end cannot be avoided but at least we can increase the depletion time to some extent. Hence following smart energy utilization strategies wastage of petroleum energy can be avoided and more oil can be drilled out of the abandoned wells. 